\title{
Article \\ Clean Foods: The Inhibitory Effect of Five Natural Juices on the Browning of Apple Slices during Drying
}

\author{
Anna Hubackova and Jan Banout* \\ Department of Sustainable Technologies, Faculty of Tropical AgriSciences, Czech University of Life Sciences, \\ Prague 16521, Czech Republic; a.hubackova@gmail.com \\ * Correspondence: banout@ftz.czu.cz; Tel.: +420-224-383-415; Fax: +420-234-381-829
}

Academic Editors: Varit Srilaong, Mantana Buanong, Chalermchai Wongs-Aree, Sirichai Kanlayanarat and Douglas D. Archbold

Received: 7 December 2015; Accepted: 28 June 2016; Published: 30 December 2016

\begin{abstract}
Enzymatic browning, which accompanies food preservation processes based on drying, is a common obstacle to obtaining marketable and consumer-appealing products. In this paper, we investigate the application of one of the methods commonly used in order to counter the browning process, namely dipping. Dipping involves a soaking of the foods in liquids or solutions in order to suppress the activity of polyphenoloxidase (PPO) enzymes, either by physically preventing oxygen from interacting with it, or by dramatically decreasing water activity and thus slowing down water-dependent reactions. In this study, juices from selected fresh fruits with high ascorbic acid content were used as natural preservatives with antibrowning effects. The juices were prepared from fruit of the following species: Sorbus aucuparia, Diospyros kaki, Hippophae rhamnoides, Actinidia deliciosa, and Rosa canina. The effect of selected juices on color change was tested on dried "Idared" apple slices and was compared to slices of freshly cut apples (standard). The browning index of all sample groups showed significant differences between treatment groups and the standard except for samples treated with Rosa canina juice. The effect of the juices was also evaluated via a sensory panel, where color change, degree of browning, change of taste, and overall acceptability of the resulting color and taste were evaluated. Results showed that the best antibrowning effect was achieved by macerate from fruits of Rosa canina. The results of this study showed that dipping in some plant juices has the potential of complementing or replacing the sulphite-based approach, which is the current method of choice of the food industry.
\end{abstract}

Keywords: natural preservants; Rosa canina; drying; browning

\section{Introduction}

Fruit is an important part of human nourishment, as it contains a number of beneficial and essential compounds, including saccharides, vitamins, minerals, fiber, and natural pigments $[1,2]$. To secure a fruit supply throughout the year, it is often necessary to preserve it [2]. One of the oldest techniques of preserving foods is by lowering the water activity in foods, for example, by drying [3]. However, through drying, fruit may lose much of its natural texture and color [4-6]. Color change is caused by both enzymatic and non-enzymatic browning [7]. One of the methods of suppressing enzymatic browning is by use of ascorbic acid [2]. Thus, the main objective of this research was to investigate the effect of natural juices with ascorbic acid for suppressing enzymatic browning of apple (Malus domestica Borkh.) slices during dehydration.

\section{Experimental Section}

Natural fruit juices of five species containing high amounts of vitamin C (Sorbus aucuparia (mountain-ash), Diospyros kaki (Japanese persimmon), Hippophae rhamnoides (sea buckthorn), 
Actinidia deliciosa (kiwifruit), Rosa canina, (dog-rose)) and a 1\% solution of ascorbic acid (AA) were used in this study. Except for the juice from Rosa canina, all juices were prepared in a juicer and diluted with an equal amount of distilled water. The juice from fruits of Rosa canina was prepared by macerating the fruits in the dark at $18{ }^{\circ} \mathrm{C}$.

Vitamin $C$ content in the juices was measured by high-performance liquid chromatography (HPLC). Samples of juices were centrifuged in 1.5-mL Eppendorf test tubes at 14,500 rpm and then filtered through a PTFE filter with a $0.45-\mu \mathrm{m}$ pore size. The samples were diluted with water at 20:1 and then analyzed via HLPC with a diode array detector (Dionex Summit, Dionex Inc., Sunnyvale, CA, USA). The chromatography conditions were as follows: isocratic elution with a mobile phase of $0.1 \%$ TFA, and a flow rate of $0.8 \mathrm{~mL} / \mathrm{min}$ on a Gemini C- $18,250 \times 4.6 \mathrm{~mm}$, a particle size of $5 \mu \mathrm{m}$ (Phenomenex, Inc., Torrance, CA, USA) column with a C-18 pre-column. Column temperature was $25^{\circ} \mathrm{C}$, and the injection volume was $20 \mu \mathrm{L}$. The program duration was $7.5 \mathrm{~min}$ with elution time of the ascorbic acid at $6.2 \mathrm{~min}$. Sample ascorbic acid content was measured at $243 \mathrm{~nm}$ and quantified by external calibration using a linear calibration for ascorbic acid of $0.004-1.000 \mathrm{mg} / \mathrm{mL}$.

A single juice or ascorbic acid solution was applied to five slices of "Idared" apple, cold stored prior to use, by soaking the slices for $10 \mathrm{~min}$. The apples were purchased at a local fruit market in Prague, Czech Republic. The treated apple slices, as well as untreated slices, were then dried in a home fruit dryer. The dryer temperature was set to $57^{\circ} \mathrm{C}$ with a constant air flow rate of $1.0 \mathrm{~m} / \mathrm{s}$. The drying was continued until a constant mass was obtained.

After drying, the degree of browning and the color change were measured on a colorimeter MiniScan ${ }^{\circledR}$ XE Plus. The results were reported using the $L^{*}-a^{*}-b^{*}$ system. Freshly cut apples were measured as a standard, and untreated dried samples measured as a control. After that, the samples treated with the fruit juices and $1 \%$ ascorbic acid were measured.

Parameters such as browning index $(B I)$, hue angle $\left(\mathrm{h}^{\circ}\right)$, and saturation index $\left(\mathrm{C}^{*}\right)$ were calculated. The $B I$ represents purity of brown color and is generally accepted as a significant parameter in color change. It is calculated as [8]

$$
B I=\frac{100(x-0.31)}{0.17} x=\frac{a+1.75 L}{5.645 L+a-3.012 b}
$$

The $B I$ assumes values between 0 and 100, where 0 corresponds to white and 100 corresponds to black. Hue angle $\left(\mathrm{h}^{\circ}\right)$ is defined as the angle between the hypotenuse and $0^{\circ}$ on the $a^{*}$ (bluish-green/red-purple) axis, and is calculated from the arctangent of $b^{*} / a^{*}$. The hue angle value corresponds to whether the object is red, orange, yellow, green, blue, or violet. Saturation index $C^{*}$ represents the hypotenuse of a right triangle created by joining points $(0,0),\left(a^{*}, b^{*}\right)$, and $\left(a^{*}, 0\right)$. The greater the $C^{*}$ value, the purer the color appears. The total color difference $\Delta E^{*}$ was calculated as $\Delta E^{*}=\left(\Delta L^{*} 2+\Delta a^{*} 2+\Delta b^{*} 2\right) 1 / 2$.

Besides colorimetry, the organoleptic properties of the apple slices were evaluated by 14 trained panelists. During panel tasting, participants filled out a questionnaire about the perceived color change, degree of browning, taste, and overall attractiveness of presented dried apples. The degree of browning and color change was evaluated in comparison with a standard of freshly cut apples. All tests were done in triplicate.

\section{Results and Discussion}

\subsection{Ascorbic Acid Content in Juices}

Ascorbic acid content was determined for all juices by HPLC except for Diospyros kaki, where this method failed. The average amount of AA in juice from Diospyros kaki was taken from the literature [9]. The highest amount of AA was found in the juice from Sorbus aucuparia and Hippophae rhamnoides (Table 1). About one third that amount was found in the juice from Rosa canina and Actinidia deliciosa. 
Table 1. Ascorbic acid content in selected fruit juices. Values are mean \pm standard deviation.

\begin{tabular}{cc}
\hline Natural Juices & Ascorbic Acid $(\mathbf{m g} / \mathbf{m L})$ \\
\hline Sorbus aucuparia & $1.79 \pm 0.20$ \\
Diospyros kaki & $0.43 \pm 0.08$ \\
Hippophae rhamnoides & $1.65 \pm 0.23$ \\
Actinidia deliciosa & $0.50 \pm 0.08$ \\
Rosa canina & $0.57 \pm 0.22$ \\
\hline
\end{tabular}

\subsection{Colorimetric Measurements}

Color is one of the most important parameters that determine the quality of raw materials and processed products. This characteristic affects consumer acceptability of the product [10]. The process of drying caused significant changes in $L^{*}, a^{*}$ and $b^{*}$ values compared to the standard (Table 2). The freshly cut standard had values indicating a light shade of green and yellow, similar to results described by Vega-Gálvez et al. [11]. According to the $\Delta E^{*}$ values (or total color difference), the samples treated with Rosa canina were the closest (smallest $\Delta E^{*}$ ) to the standard in color. In contrast, the Sorbus aucuparia-treated samples showed the greatest difference. Furthermore, none of the treatments achieved a greener shade than the standard. All of the treatments showed an increase in the yellow color component with the most prominent difference measured with Sorbus aucuparia samples. The smallest difference in the yellow component was found with the Hippophae rhamnoides and Actinidia deliciosa samples. A small amount of blue color was found with the ascorbic acid and Rosa canina samples.

Table 2. Colorimetric data for freshly cut apple slices (standard), and samples treated with natural juices, ascorbic acid (AA) solution, or left untreated. Values are mean \pm standard deviation.

\begin{tabular}{|c|c|c|c|c|c|c|c|}
\hline Treatments & $L^{*}$ & $a^{*}$ & $b^{*}$ & $\Delta L^{* \mathrm{z}}$ & $\Delta a^{* \mathrm{y}}$ & $\Delta b^{* x}$ & $\Delta E^{* \mathrm{w}}$ \\
\hline Standard & $86.78 \pm 0.98$ & $-0.29 \pm 0.33$ & $25.71 \pm 1.37$ & & & & \\
\hline Sorbus aucuparia & $82.08 \pm 2.50$ & $7.27 \pm 2.02$ & $40.98 \pm 3.11$ & $-4.70 \pm 2.50$ & $7.56 \pm 2.02$ & $15.27 \pm 2.11$ & $18.11 \pm 2.79$ \\
\hline Diospyros kaki & $80.46 \pm 1.28$ & $5.56 \pm 0.98$ & $31.44 \pm 1.10$ & $-6.32 \pm 2.55$ & $5.85 \pm 1.88$ & $5.73 \pm 2.46$ & $10.52 \pm 2.89$ \\
\hline Hippophae rhamnoides & $77.48 \pm 2.21$ & $7.82 \pm 2.19$ & $27.15 \pm 2.22$ & $-9.30 \pm 2.21$ & $8.11 \pm 2.19$ & $1.44 \pm 2.22$ & $13.15 \pm 2.92$ \\
\hline Actinidia deliciosa & $79.34 \pm 1.54$ & $6.21 \pm 2.62$ & $27.46 \pm 2.35$ & $-7.44 \pm 2.54$ & $6.50 \pm 2.62$ & $1.75 \pm 2.35$ & $10.82 \pm 2.47$ \\
\hline Rosa canina & $84.29 \pm 2.77$ & $4.41 \pm 1.91$ & $23.18 \pm 2.03$ & $-2.05 \pm 2.77$ & $4.70 \pm 1.91$ & $-2.53 \pm 2.03$ & $8.51 \pm 2.02$ \\
\hline $1 \%$ solution of $\mathrm{AA}$ & $74.09 \pm 1.71$ & $11.56 \pm 2.21$ & $25.28 \pm 1.85$ & $-12.7 \pm 1.71$ & $11.85 \pm 2.21$ & $-0.43 \pm 1.85$ & $17.76 \pm 2.04$ \\
\hline Untreated samples & $77.78 \pm 2.72$ & $8.19 \pm 2.11$ & $31.89 \pm 2.07$ & $9.00 \pm 2.72$ & $8.48 \pm 2.11$ & $6.18 \pm 2.07$ & $13.98 \pm 2.82$ \\
\hline
\end{tabular}

\subsection{Hue Angle, Saturation, and the Browning Index}

Values of $L^{*}, a^{*}$, and $b^{*}$ (Table 2) were used for the calculation of $\mathrm{h}^{\circ}, \mathrm{C}^{*}$, and BI (Table 3). According to the results of analysis of variance of BI (F-test, $p \leq 0.005$ ), there were statistically significant differences between the means of BI of the different treatments. The Box-and-Whisker plot (Figure 1) shows the specific differences among the treatments. It is obvious that only the Rosa canina treatment approached the standard in quality. These results are in agreement with Zocca et al. [12], where there was significant inhibition by Rosa canina extracts against polyphenol oxidase (PPO). The highest degree of browning was observed with apples treated with juices from Sorbus aucuparia. Based on $\mathrm{h}^{\circ}$ and $\mathrm{C}^{*}$, the closest values to the standard were also found with apple slices treated with Rosa canina juice. 
Table 3. Hue angle $\left(\mathrm{h}^{\circ}\right)$, saturation index $\left(\mathrm{C}^{*}\right)$, and browning index $(\mathrm{BI})$ of apple slices treated with selected fruit juices, ascorbic acid (AA), or left untreated. Values are mean \pm standard deviation.

\begin{tabular}{cccc}
\hline Treatments & $\mathbf{h}^{\circ}$ & $\mathbf{C}^{*}$ & BI \\
\hline Standard & $90.65 \pm 3.02$ & $25.71 \pm 2.23$ & $33.59 \pm 2.36$ \\
Sorbus aucuparia & $79.94 \pm 2.36$ & $41.62 \pm 4.36$ & $73.98 \pm 3.93$ \\
Diospyros kaki & $79.98 \pm 3.47$ & $31.93 \pm 2.14$ & $54.16 \pm 4.45$ \\
Hippophae rhamnoides & $73.93 \pm 4.02$ & $28.25 \pm 1.89$ & $51.08 \pm 6.35$ \\
Actinidia deliciosa & $77.26 \pm 3.69$ & $28.15 \pm 1.62$ & $48.58 \pm 4.03$ \\
Rosa canina & $79.23 \pm 2.78$ & $23.60 \pm 1.44$ & $36.66 \pm 8.11$ \\
1\% solution of AA & $65.42 \pm 3.56$ & $27.80 \pm 2.40$ & $53.37 \pm 4.89$ \\
Untreated samples & $75.60 \pm 4.25$ & $32.93 \pm 3.32$ & $60.06 \pm 3.97$ \\
\hline
\end{tabular}

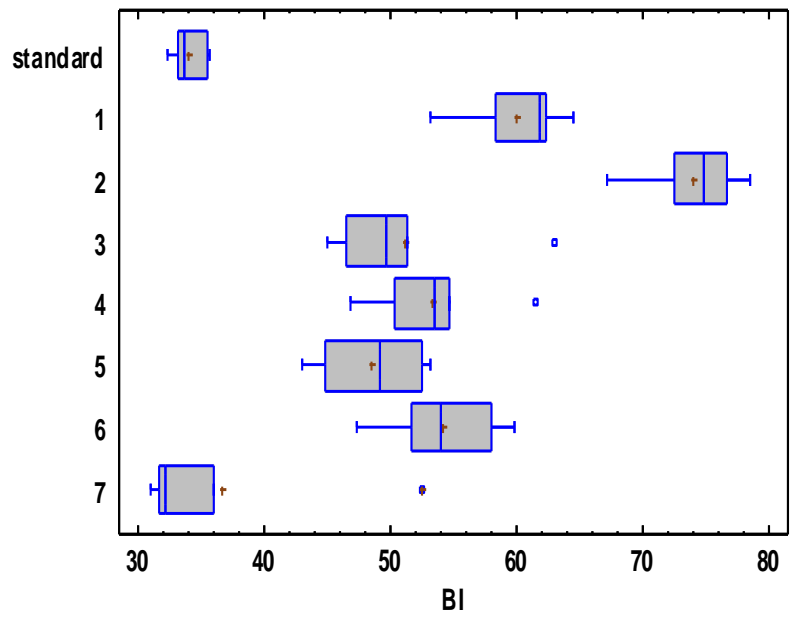

Figure 1. Box-and-Whisker Plot. Y-axis: $1=$ untreated samples; 2 = Sorbus aucuparia; $3=$ Hippophae rhamnoides; $4=1 \%$ solution of AA; $5=$ Actinidia deliciosa; $6=$ Diospyros kaki; and $7=$ Rosa canina $. \mathrm{BI}=$ browning index.

\subsection{Organoleptic Analyses}

Consumer sensory analyses revealed that perceived color change, compared with the standard, was rated as "moderate" for samples treated with Rosa canina juice and ascorbic acid solution (Table 4). In the samples treated with Actinidia deliciosa, Hippophae rhamnoides, and Diospyros kaki juices, as well as the untreated samples, color change was rated as "medium," and, for samples treated with Sorbus aucuparia, color change was "significant."

Table 4. Sensory evaluation results of apple slices treated with selected fruit juices, ascorbic acid (AA), or left untreated. Values are mean \pm standard deviation.

\begin{tabular}{cccc}
\hline Treatment & Color Change versus Standard & Browning versus Standard & Overall Appearance \\
\hline Sorbus aucuparia & $2.6 \pm 0.50^{\mathrm{z}}$ & $2.1 \pm 0.86$ & $2.1 \pm 0.36^{\mathrm{y}}$ \\
Diospyros kaki & $2.3 \pm 0.61$ & $2.3 \pm 0.61$ & $2.0 \pm 0.28$ \\
Hippophae rhamnoides & $2.1 \pm 0.95$ & $3.0 \pm 0.25$ & $2.6 \pm 0.42$ \\
Actinidia deliciosa & $1.9 \pm 0.47$ & $2.0 \pm 0.39$ & $1.9 \pm 0.31$ \\
Rosa canina & $1.0 \pm 0.10$ & $1.0 \pm 0.16$ & $1.8 \pm 0.14$ \\
1\% solution of AA & $1.3 \pm 0.36$ & $1.6 \pm 0.51$ & $1.9 \pm 0.41$ \\
Untreated samples & $2.3 \pm 0.61$ & $1.8 \pm 0.7$ & $1.7 \pm 0.37$ \\
\hline
\end{tabular}

${ }^{\mathrm{z}}$ Values for color change compared with the standard and browning compared with the standard: 1 = moderate; 2 = medium; 3 = significant; ${ }^{y}$ Overall appearance: 1 = very good; 2 = satisfactory; and 3 = unacceptable.

Samples treated with Rosa canina juice displayed the lowest degree of browning compared to the standard, while there was a "medium" degree of browning with the AA solution, with juice 
from Actinidia deliciosa, Sorbus aucuparia, and Diospyros kaki, and with those that were left untreated. The greatest browning was observed in the Hippophae rhamnoides samples.

Overall appearance was not compared with the standard; rather, it focused on the overall acceptability by the consumer. The best appearances were given to samples treated with $1 \%$ AA solution, Rosa canina juice, and to the untreated samples, while samples treated with Actinidia deliciosa, Diospyros kaki, and Sorbus aucuparia exhibited a "satisfactory" appearance. Samples treated with juice from Hippophae rhamnoides averaged between "satisfactory" and "unacceptable".

The fourth aspect evaluated via sensory analysis was taste, specifically sweetness, bitterness, and sourness. The least sweet were the Rosa canina-treated samples (Table 5). A sweet taste was more pronounced in the samples treated with juices from Hippophae rhamnoides, Diospyros kaki, Actinidia deliciosa, and the AA solution. Untreated samples were rated as the sweetest. Most samples showed only a "mild" degree of bitterness; only Sorbus aucuparia caused the samples to be rated as "medium" with regard to bitterness. Sourness was the least pronounced in samples treated with Diospyros kaki juice and in untreated samples. Samples treated with Actinidia deliciosa, Hippophae rhamnoides, Sorbus aucuparia, and Rosa canina juices showed a "medium" degree of sourness, whereas those treated with the AA solution were rated as the most sour.

Table 5. Degustation of apple slices treated with selected fruit juices, ascorbic acid (AA), or left untreated. Values are mean \pm standard deviation.

\begin{tabular}{ccccc}
\hline Treatment & Sweetness & Bitterness & Sourness & Overall Flavor \\
\hline Sorbus aucuparia & $1.8 \pm 0.16^{\mathrm{z}}$ & $1.5 \pm 0.25$ & $2.1 \pm 0.15$ & $2.3 \pm 0.34 \mathrm{y}$ \\
Diospyros kaki & $2.0 \pm 0.32$ & $1.3 \pm 0.47$ & $1.4 \pm 0.63$ & $2.0 \pm 0.36$ \\
Hippophae rhamnoides & $1.9 \pm 0.24$ & $1.4 \pm 0.50$ & $1.8 \pm 0.40$ & $1.5 \pm 0.41$ \\
Actinidia deliciosa & $2.0 \pm 0.47$ & $1.3 \pm 0.44$ & $1.6 \pm 0.46$ & $2.1 \pm 0.23$ \\
Rosa canina & $1.5 \pm 0.33$ & $1.3 \pm 0.37$ & $2.1 \pm 0.64$ & $1.8 \pm 0.34$ \\
1\% solution of AA & $1.6 \pm 0.26$ & $1.4 \pm 0.63$ & $2.6 \pm 0.30$ & $2.3 \pm 0.52$ \\
Untreated apples & $2.1 \pm 0.28$ & $1.1 \pm 0.36$ & $1.5 \pm 0.26$ & $2.0 \pm 0.46$ \\
\hline
\end{tabular}

z Sweetness, bitterness and sourness values: 1 = mild; 2 = medium; 3 = significant; ${ }^{y}$ Overall flavor: very good, satisfactory, unacceptable.

Samples treated with the juice from Hippophae rhamnoides achieved the highest rating for overall flavor. The Rosa canina, Diospyros kaki, Actinidia deliciosa, Sorbus aucuparia, ascorbic acid, and untreated samples received mostly "satisfactory" ratings.

\section{Conclusions}

In this study, the influence of selected fruit juices on enzymatic browning in dried apple slices was evaluated. Those treated with a diluted Rosa canina juice showed the smallest total color difference and the lowest degree of browning, as determined from colorimetric data. The sensory evaluation data suggested that using the Rosa canina extract resulted in a product possessing the most appealing organoleptic properties. We conclude that, based on this study, using plant-based food preservatives is a viable and efficient alternative to the currently used techniques.

Acknowledgments: This research was supported by the Internal Grant Agency of the Faculty of Tropical AgriSciences, Czech University of Life Sciences Prague, project no. 20155017.

Author Contributions: Anna Hubackova-experimental procedure and measurements, data collection and analyses, writing of the manuscript. Jan Banout-experimental design and research management, data evaluation, contribution to manuscript preparation mainly the chapter Results and Discussion, ensuring financial sources for the research.

Conflicts of Interest: The authors declare no conflicts of interest. 


\section{References}

1. Nour, V.; Trandafir, I.; Ionica, M.E. Compositional characteristics of fruits of several apple (Malus domestica borkh.) cultivars. Not. Bot. Hortic. Agrobot. Cluj 2010, 38, 228-233.

2. Rahman, M.S. Handbook of Food Preservation; CRC Press: Boca Raton, FL, USA, 2007.

3. Prvulovic, S.; Tolmac, D. Convection Drying in the Food Industry; International Commission of Agricultural Engineering: Beograd, Serbia, 2007.

4. Pilližota, V.; Šubarić, D. Control of enzymatic browning of foods. Food Technol. Biotechnol. 1998, 36, 219-227.

5. Raymond, G.G. Reporting of objective color measurements. HortScience 1992, 27, 1254-1255.

6. Lozano, J.E. Fruit Manufacturing: Scientific Basis, Engineering Properties and Deteriorative Reactions of Technological Importance; Washington State University: Bahia Blanca, Argentina, 2006.

7. Özglu, H.; Bayindirli, A. Inhibition of enzymic browning in cloudy apple juice with selected antibrowning agents. Food Control 2002, 13, 213-221. [CrossRef]

8. Mohammad, A.; Rafiee, S.; Emam-Djomeh, Z.; Keyhani, A. Kinetic models for color changes in kiwifruit slices during hot air drying. World J. Agric. Sci. 2008, 4, 376-383.

9. Souza, E.L.; Souza, A.L.K.; Tiecher, A.; Girardic, C.L.; Nora, L.; da Silva, J.A.; Argenta, L.C.; Rombaldi, C.V. Changes in enzymatic activity, accumulation of proteins and softening of persimmon (Diospyros kaki Thunb.) flesh as a function of pre-cooling acclimatization. Sci. Hortic. 2011, 127, 242-248. [CrossRef]

10. Fijalkowska, A.; Nowacka, M.; Wiktor, A.; Sledz, M.; Witrowa-Rajcher, D. Ultrasound as a pretreatment method to improve drying kinetics and sensory properties of dried apple. J. Food Proc. Eng. 2015. [CrossRef]

11. Vega-Gálvez, A.; Ah-Hen, K.; Chacana, M.; Vergara, J.; Martínez-Monzó, J.; García-Segovia, P.; Lemus-Mondaca, R.; Scala, K. Effect of temperature and air velocity on drying kinetics, antioxidant capacity, total phenolic content, color, texture and microstructure of apple (var. Granny Smith) slices. Food Chem. 2012, 132, 51-59. [CrossRef] [PubMed]

12. Zocca, F.; Lomolino, G.; Lante, A. Dog rose and pomegranate extracts as agents to control enzymatic browning. Food Res. Int. 2011, 44, 957-963. [CrossRef]

(C) 2016 by the authors; licensee MDPI, Basel, Switzerland. This article is an open access article distributed under the terms and conditions of the Creative Commons Attribution (CC-BY) license (http://creativecommons.org/licenses/by/4.0/). 